\title{
A PREVENÇÃO DO HIV / AIDS SEGUNDO O OLHAR DAS MULHERES: 0 CASO DO GRUPO DE DIREITOS REPRODUTIVOS EM J UIZ DE FORA / MGa
}

\author{
HIV/ AIDS prevention according to womens view: \\ The reproductive rights group case in J uiz de Fora / MG, Brazil \\ La prevencíon del HIV/ SIDA según el punto de vista de las mujeres: \\ El caso del grupo de derechos reproductivos en J uiz de Fora / MG, Brasil
}

Eenir Pereirade Paiva

Meria Catarina Salvador da Matta

\begin{abstract}
Oestudo teve com dojeto as práticas de prevenção do HV na visão de milheres participantes do Gupo de Direitos Reprodutivos ${ }^{1}$ pois, apesar das diversas camparhes dirigidas à prevenção, as estáísticas indicamaumento significativo demilheres infectadas pelo HV. Os dbjetivos foram 1) identificar as informaçães que as participantes do Gupo de Diretos Reprodutivos possuemsolore as formas de prevenção contraHV/ADS; 2) identificar estratégias de prevenção contrao HV/ADS queas milheres db Gupo de Diretos Reprodu tivos adbtamemsua práticasexul; 3) analisar as formas de prevenção contra o HV/ADS addadas pelas milheres do Gupo deDireitos Reprodutivos no Departamento de Urgênciae Emergêndia Norte(DEV) em/uiz deFora MG Mteodblogia: privilegiarse a pesquisa qualitativa, sob a forma de estudo de caso², oriundas do grupo de discussão mencionado. Para coleta de dados, empregou-seaentrevistasemiestruturada, juntamentecomobservação participante comregistroemdário decampo. Os resultados sugeremqueas participantes aindanantêmpenplexidades quantoàs formas detransmissãodo $\mathrm{HV}$, prosseguemrepresentandb aADS


dos parceiros no que concerme ao uso do mesmo. Ognupo refere não adbtar métodos prevertivos de forma contínua. Teorias de aprendizagen foramanalisadas como dbjetivo de corhecer emqual tipo de aprendiz estanos trabalhando. Ao final apresentamse algumas considerações esugestões, tendb emvista tomar nais efetives as ações educatives nesta área ca saúde púdica.
\end{abstract}

Palaurasdhave ADS. Prevenção de Doenças Transmissíveis. Educação. Milheres.

The study foa uses the representations of female participants of the Reproductive Rights Group about HV prevention pradices, because, in spite of several compaigns directed to prevertion, stdistics showasignificant increasein then unber of $H$ Vinfected nomen The objectives were: 1) to identify the knowedge about HV/ADS prevertion methods that took part of the Reproductive Rights Group possessed; 2) to identify prevertion strategies against HV/ADS that those women use in their sexd life, 3) to analyze the prevertion methods adopted by Reproductive Rights Group at Energency and Urgency North Departmert (DEN) in Juiz deFora, Mnas Cerais, Brazil. Methodblogy. we wrivileged the qualitativeresearch, intheformof acasestudy, deriving from the disassion group mentioned. To collect data, we used a semistructured interview, associating with participant obsenvetion with field daybook record. The results suggest that the participants are sill perplexed about HV transmitting forms, and they keep represerting ADS as an inarable and dangerous dseese and in spite of giving value to the use of condons as a preventive medharism, they find resistance from their partners to use it The grap reported not to use preventive mesures continuausly. Leamingtheories ${ }^{5}$ was analized with the purpose to knowin wich type of apretice weare workwith Finally, someconsidertions and suggestions are made, aiming at making the eductional actions in this public health areanore effective

\section{Keyouds}

ADS Commricade Dseese Prevertion. Eduction Vorren
B estudotuno corro dijetivo lás prádicas del HVsegínla visiónde mijeres participantes del Grupo de Derechos Reproductivos, pues, a pesardelas dversas canpãnesdingidesalaprevenjón, lasestadsticas indican un auretosignificativo demejeres infectadas por $\mathrm{H} \mathrm{HV}$. Los dgietivosfueron idantificar lasinformaciones quelas participantes da Gupo de Derechos Reproductivos poseen sobre las formas de prevenióncortrae $\mathrm{HV}$, idantificarestraégias deprevencóncortrae HVquelas mijeres da Gupo de Derechos Peprodutivos adpptanen su prádica sexal; Analisar las formas de prevención contra el HV adbptadas por las mijeres dal Gupo de Derechos Reproductivos en

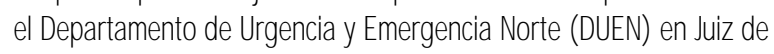
Fora, Mnas Gerais, Brasil. Metodblogía: se dio prioridad a la investigación a litativa, bajo la forma deestudo de caso originarias del grypodedisasiónmencionadb. Paralacolecióndelos datos, se usó la entrevista semi-estructurada, junto con la obsenvación participartecomregistroendariodecampo. Los resultadbssugieren quelas partiapartes tocavia semartienen pendejas en lo referertea las formas de transmisón de $H V$, continuen representandb a SDA comounaenfermedadpeligrosaeinaradley, adespedhodevelorizar el presentivoconomecarisnodeprevendón enartranresistenda de sus perejas en lo que condemeal uso de los mismos. I gnpo refieremoadoptar métodos prevertivos deformacontín aprendzado fueron andizadas con el djetivo de conocer comqué tipo de aprendz estanos trabajandb. A find se presentan algunes considaraconesysugerendas, teriendbenarita hacer más efectives las acciones eductives en esta áreadela salud pública

\section{Palabas dave}

SIDA Prevenión de Efermedades Transmisibles. Eduracoón Mjeres. 
1. VilleaWDiniz S Aepidemia da ADS entre as milheres. NPADS/ GFSS São Paulo: 1998.

2. Ludke M André MEA Pesquisa emEducação: abordagens qualitatives. São Paulo. ĐU, 1986.

3. Mrayo MCS. Odesafio do conhedimento: pesquisa qualitativaem saúde Rio deJaneiro / São Paulo: Abrasco / Huitec, 1994.

\section{Nota}

a Dissetação de Mestradb defendida emjulho de 2003 ra Escola de EnfermagemAma Nery/ UFR.

Mesters Degree Dissettaion defended injuly of 2003 in the Ama Nery School of Nursing/ UPJ.

Dissertacón del Mester defendida en jule de 2003 en la Esaula de Eremería Ama/Nery/ URI.
4. SalgadbZ Arelaçãosocial coñ atitudepreventivano contexto do HV/ALS: U ha perspectiva paraazssistênciadesaúdefundamentada emAlfred Schuz. Rio de Janeiro:2000. Tese[Doutorado]. Rio de Janeiro: Escola de EfremagemAma Nery, 133 p.

5. Mbreira MA Ensino eaprendzagem enfoques teónicos - Skimer, Gagné, Bruner, Piaget, Ausubel eRogers. São Paulo: EditoraMbraes, 1985.

\section{Sobre as Autoras}

\section{Eeri Peréa de Paía}

Aluna do Curso de Doutorado em Enfermagem da Escola de EnfermagemAmaNery (EAN) / UFP).

email: elenirbolpato@ychoo.combr

\section{Maria Graina Salhador da Motta}

Professora Adjunta e pesquisadora do Núdleo de Pesquisa Enfermageme Saúde Coletiva do Departamento de Enfermagemde Saúde Púdica da $\pm A N /$ UPJ.

email: mesmotta@ubl.combr 